\title{
ON TZITZEICA CURVES IN EUCLIDEAN 3-SPACE $\mathbb{E}^{3}$
}

\author{
Bengü Bayram, Emrah Tunç, Kadri Arslan and Günay Öztürk
}

\begin{abstract}
In this study, we consider Tzitzeica curves (Tz-curves) in a Euclidean 3space $\mathbb{E}^{3}$. We characterize such curves according to their curvatures. We show that there is no Tz-curve with constant curvatures (W-curves). We consider Salkowski (TCcurve) and anti-Salkowski curves.
\end{abstract}

Keywords: Tz-curves, W-curves, TC-curves

\section{Introduction}

Gheorgha Tzitzeica, a Romanian mathematician (1872-1939), introduced a class of curves, nowadays called Tzitzeica curves, and a class of surfaces of the Euclidean 3 -space called Tzitzeica surfaces. A Tzitzeica curve in $\mathbb{E}^{3}$ is a spatial curve $x=x(s)$ for which the ratio of its torsion $\kappa_{2}$ and the square of the distance $d_{o s c}$ from the origin to the osculating plane at an arbitrary point $x(s)$ of the curve is constant, i.e.,

$$
\frac{\kappa_{2}}{d_{o s c}^{2}}=a
$$

where $d_{o s c}=\left\langle N_{2}, x\right\rangle$ and $a \neq 0$ is a real constant, $N_{2}$ is the binormal vector of $x$.

In [3] the authors gave the connections between the Tzitzeica curve and the Tzitzeica surface in a Minkowski 3-space and the original ones from the Euclidean 3 -space. In [7] the authors determined the elliptic and hyperbolic cylindrical curves satisfying Tzitzeica condition in a Euclidean space. In [12], the elliptic cylindrical curves verifying Tzitzeica condition were adapted to the Minkowski 3-space. In [2], the authors gave the necessary and sufficient condition for a space curve to become a Tzitzeica curve. The new classes of symmetry reductions for the Tzitzeica curve equation were determined. In [1], the authors were interested in the curves of Tzitzeica type and they investigated the conditions for non-null general helices, pseudo-spherical curves and pseudo-spherical general helices to become of Tzitzeica type in a Minkowski space $\mathbb{E}_{1}^{3}$.

Received December 19, 2017; accepted June 20, 2018

2010 Mathematics Subject Classification. Primary 53A04; Secondary 53A05 
A Tzitzeica surface in $\mathbb{E}^{3}$ is a spatial surface $M$ given with the parametrization $X(u, v)$ for which the ratio of its Gaussian curvature $K$ and the distance $d_{\text {tan }}$ from the origin to the tangent plane at any arbitrary point of the surface is constant, i.e.,

$$
\frac{K}{d_{\tan }^{4}}=a_{1}
$$

for a constant $a_{1}$. The orthogonal distance from the origin to the tangent plane is defined by

$$
d_{\tan }=\langle X, \vec{U}\rangle
$$

where $X$ is the position vector of the surface and $\vec{U}$ is a unit normal vector of the surface.

The asymptotic lines of a Tzitzeica surface with a negative Gausssian curvature are Tzitzeica curves [7]. In [18], the authors gave the necessary and sufficient condition for the Cobb-Douglas production hypersurface to be a Tzitzeica hypersurface. In addition, a new Tzitzeica hypersurface was obtained in parametric, implicit and explicit forms in [8]

In this study, we consider Tzitzeica curves (Tz-curves) in a Euclidean 3-space $\mathbb{E}^{3}$. Furthermore, we investigate a Tzitzeica curve in a Euclidean 3 -space $\mathbb{E}^{3}$ whose position vector $x=x(s)$ satisfies the parametric equation

$$
x(s)=m_{0}(s) T(s)+m_{1}(s) N_{1}(s)+m_{2}(s) N_{2}(s),
$$

for some differentiable functions, $m_{i}(s), 0 \leq i \leq 2$, where $\left\{T, N_{1}, N_{2}\right\}$ is the Frenet frame of $x$. We characterize such curves according to their curvatures. We show that there is no Tzitzeica curve in $\mathbb{E}^{3}$ with constant curvatures (W-curves). We give the relations between the curvatures of the Tz-Salkowski curve (TC-curve) and the Tz-anti-Salkowski curve.

\section{Basic Notations}

Let $x: I \subset \mathbb{R} \rightarrow \mathbb{E}^{3}$ be a unit speed curve in a Euclidean 3-space $\mathbb{E}^{3}$. Let us denote $T(s)=x^{\prime}(s)$ and call $T(s)$ a unit tangent vector of $x$ at $s$. We denote the curvature of $x$ by $\kappa_{1}(s)=\left\|x^{\prime \prime}(s)\right\|$. If $\kappa_{1}(s) \neq 0$, then the unit principal normal vector $N_{1}(s)$ of the curve $x$ at $s$ is given by $x^{\prime \prime}(s)=\kappa_{1}(s) N_{1}(s)$. The unit vector $N_{2}(s)=T(s) \times N_{1}(s)$ is called the unit binormal vector of $x$ at $s$. Then we have the Serret-Frenet formulae:

$$
\begin{aligned}
T^{\prime}(s) & =\kappa_{1}(s) N_{1}(s), \\
N_{1}^{\prime}(s) & =-\kappa_{1}(s) T(s)+\kappa_{2}(s) N_{2}(s), \\
N_{2}^{\prime}(s) & =-\kappa_{2}(s) N_{1}(s)
\end{aligned}
$$

where $\kappa_{2}(s)$ is the torsion of the curve $x$ at $s$ (see, [10]). 
If the Frenet curvature $\kappa_{1}(s)$ and torsion $\kappa_{2}(s)$ of $x$ are constant functions then $x$ is called a screw line or a helix [9]. Since these curves are the traces of 1-parameter family of the groups of Euclidean transformations then F. Klein and S. Lie called them $W$-curves [14]. It is known that a curve $x$ in $\mathbb{E}^{3}$ is called a general helix if the ratio $\kappa_{2}(s) / \kappa_{1}(s)$ is a nonzero constant [16]. Salkowski (resp. anti-Salkowski) curves in a Euclidean space $\mathbb{E}^{3}$ are generally known as the family of curves with A constant curvature (resp. torsion) but non-constant torsion (resp. curvature) with an explicit parametrization [15, 17] (for T.C-curve see also [13]).

For a space curve $x: I \subset \mathbb{R} \rightarrow \mathbb{E}^{3}$, the planes at each point of $x(s)$ spanned by $\left\{T, N_{1}\right\},\left\{T, N_{2}\right\}$ and $\left\{N_{1}, N_{2}\right\}$ are known as the osculating plane, the rectifying plane and normal plane, respectively. If the position vector $x$ lies on its rectifying plane, then $x(s)$ is called rectifying curve [5]. Similarly, the curve for which the position vector $x$ always lies in its osculating plane is called osculating curve. Finally, $x$ is called normal curve if its position vector $x$ lies in its normal plane.

Rectifying curves characterized by the simple equation

$$
x(s)=\lambda(s) T(s)+\mu(s) N_{2}(s),
$$

where $\lambda(s)$ and $\mu(s)$ are smooth functions and $T(s)$ and $N_{2}(s)$ are tangent and binormal vector fields of $x$, respectively $[5,6]$.

For a regular curve $x(s)$, the position vector $x$ can be decomposed into its tangential and normal components at each point:

$$
x=x^{T}+x^{N} .
$$

A curve in $\mathbb{E}^{3}$ is called $N$-constant if the normal component $x^{N}$ of its position vector $x$ is of constant length $[4,11]$. It is known that a curve in $\mathbb{E}^{3}$ is congruent to an $N$-constant curve if and only if the ratio $\frac{\kappa_{2}}{\kappa_{1}}$ is a non-constant linear function of an arc-length function $s$, i.e., $\frac{\kappa_{2}}{\kappa_{1}}(s)=c_{1} s+c_{2}$ for some constants $c_{1}$ and $c_{2}$ with $c_{1} \neq 0$ [4]. Further, an $N$-constant curve $x$ is called first kind if $\left\|x^{N}\right\|=0$, otherwise second kind [11].

\section{Tzitzeica Curves in $\mathbb{E}^{3}$}

In the present section we characterize Tzitzeica curves in $\mathbb{E}^{3}$ in terms of their curvatures.

Definition 3.1. Let $x: I \subset \mathbb{R} \rightarrow \mathbb{E}^{3}$ be a unit speed curve with curvatures $\kappa_{1}(s)>0$ and $\kappa_{2}(s) \neq 0$. If the torsion of $x$ satisfies the condition

$$
\kappa_{2}(s)=a \cdot d_{o s c}^{2},
$$

for some real constant $a$ then $x$ is called Tzitzeica curve (Tz-curve), where

$$
d_{o s c}=\left\langle N_{2}, x\right\rangle
$$

is the orthogonal distance from the origin to the osculating plane of $x$. 
We have the following result.

Proposition 3.1. Let $x: I \subset \mathbb{R} \rightarrow \mathbb{E}^{3}$ be a unit speed curve in $\mathbb{E}^{3}$. If $x$ is a Tz-curve, then the equation

$$
\kappa_{2}^{\prime}\left\langle x, N_{2}\right\rangle+2 \kappa_{2}^{2}\left\langle x, N_{1}\right\rangle=0
$$

holds.

Proof. Let $x$ be a unit speed curve in $\mathbb{E}^{3}$, then by the use of the equations (3.1) and (3.2) we get

$$
\frac{\kappa_{2}(s)}{\left\langle N_{2}, x\right\rangle^{2}}=a \neq 0 .
$$

Further, differentiating the equation (3.4), we obtain the result.

Definition 3.2. Let $x: I \subset \mathbb{R} \rightarrow \mathbb{E}^{3}$ be a unit speed curve with curvatures $\kappa_{1}(s)>0$ and $\kappa_{2}(s) \neq 0$. Then $x$ is a spherical curve if and only if

$$
\frac{\kappa_{2}(s)}{\kappa_{1}(s)}=\left(\frac{\kappa_{1}^{\prime}(s)}{\kappa_{2}(s) \kappa_{1}^{2}(s)}\right)^{\prime}
$$

holds [9].

Theorem 3.1. Let $x: I \subset \mathbb{R} \rightarrow \mathbb{E}^{3}$ be a unit speed spherical curve in $\mathbb{E}^{3}$. If $x$ is a Tz-curve then the equation

$$
\frac{\kappa_{2}^{\prime}(s)}{2 \kappa_{2}^{3}(s)}=\frac{\kappa_{1}(s)}{\kappa_{1}^{\prime}(s)}
$$

holds between the curvatures of $x$.

Proof. Let $x$ be a unit speed spherical curve in $\mathbb{E}^{3}$. Then we have

$$
\|x\|=r
$$

where $r$ is the radius of the sphere. Differentiating the equation (3.7) with respect to $s$, we get

$$
\langle x, T\rangle=0 .
$$

Further, differentiating the equation (3.8), we have

$$
\left\langle x, N_{1}\right\rangle=-\frac{1}{\kappa_{1}} .
$$

By differentiating the equation (3.9), we obtain

$$
\left\langle x, N_{2}\right\rangle=\frac{\kappa_{1}^{\prime}}{\kappa_{1}^{2} \kappa_{2}} .
$$

Finally, substituting (3.9) and (3.10) into (3.3), we get the result. 
Corollary 3.1. Let $x: I \subset \mathbb{R} \rightarrow \mathbb{E}^{3}$ be a unit speed spherical Tz-curve in $\mathbb{E}^{3}$. Then the torsion of $x$ satisfies the equation

$$
\kappa_{2}=\sqrt{\frac{\kappa_{1}^{\prime \prime} \kappa_{1}-2\left(\kappa_{1}^{\prime}\right)^{2}}{3 \kappa_{1}^{2}}} .
$$

Proof. Substituting (3.6) into (3.5), we get the result.

Corollary 3.2. Let $x: I \subset \mathbb{R} \rightarrow \mathbb{E}^{3}$ be a unit speed anti-Salkowski spherical Tz-curve in $\mathbb{E}^{3}$. Then the curvature of $x$ is given by

$$
\kappa_{1}=\frac{\sqrt{3} \kappa_{2}}{c_{1} \sin \left(\sqrt{3} \kappa_{2} s\right)-c_{2} \cos \left(\sqrt{3} \kappa_{2} s\right)}
$$

where $c_{1}, c_{2}$ are integral constants and $\kappa_{2}$ is the constant torsion of $x$.

Proof. Let $x: I \subset \mathbb{R} \rightarrow \mathbb{E}^{3}$ be a unit speed anti-Salkowski spherical Tz-curve in $\mathbb{E}^{3}$. Then from (3.11), we obtain the differential equation

$$
\kappa_{1}^{\prime \prime} \kappa_{1}-2\left(\kappa_{1}^{\prime}\right)^{2}-3 \kappa_{1}^{2} \kappa_{2}^{2}=0
$$

which has the solution (3.12).

Lemma 3.1. Let $x: I \subset \mathbb{R} \rightarrow \mathbb{E}^{3}$ be a unit speed curve in $\mathbb{E}^{3}$ whose position vector satisfies the parametric equation

$$
x(s)=m_{0}(s) T(s)+m_{1}(s) N_{1}(s)+m_{2}(s) N_{2}(s)
$$

for some differentiable functions, $m_{i}(s), 0 \leq i \leq 2$. If $x$ is a Tz-curve then we get

$$
\begin{aligned}
m_{0}^{\prime}-\kappa_{1} m_{1} & =1, \\
m_{1}^{\prime}+\kappa_{1} m_{0}-\kappa_{2} m_{2} & =0, \\
m_{2}^{\prime}+\kappa_{2} m_{1} & =0, \\
\kappa_{2}^{\prime} m_{2}+2 \kappa_{2}^{2} m_{1} & =0 .
\end{aligned}
$$

Proof. Let $x: I \subset \mathbb{R} \rightarrow \mathbb{E}^{3}$ be a unit speed curve in $\mathbb{E}^{3}$. Then, by taking the derivative of (3.14) with respect to the parameter $s$ and using the Frenet formulae, we obtain

$$
\begin{aligned}
x^{\prime}(s)= & \left(m_{0}^{\prime}(s)-\kappa_{1}(s) m_{1}(s)\right) T(s) \\
& +\left(m_{1}^{\prime}(s)+\kappa_{1}(s) m_{0}(s)-\kappa_{2}(s) m_{2}(s)\right) N_{1}(s) \\
& +\left(m_{2}^{\prime}(s)+\kappa_{2}(s) m_{1}(s)\right) N_{2}(s) .
\end{aligned}
$$

Further, using the equations (3.3) and (3.16), we get (3.15). 
Theorem 3.2. Let $x: I \subset \mathbb{R} \rightarrow \mathbb{E}^{3}$ be a unit speed anti-Salkowski Tz-curve in $\mathbb{E}^{3}$ (with the curvatures $\kappa_{1}>0$ and $\kappa_{2} \neq 0$ ) given with the parametrization (3.14). Then $x$ is congruent to a rectifying curve with the parametrization

$$
x(s)=\left(s+c_{1}\right) T(s)+c_{2} N_{2}(s)
$$

where $c_{1}$ and $c_{2}$ are integral constants.

Proof. Let $x$ be a unit speed anti-Salkowski Tz-curve in $\mathbb{E}^{3}$. Then, the torsion $\kappa_{2}$ of $x$ is constant. From the equation (3.15), we get

$$
\begin{aligned}
& m_{0}=s+c_{1} \\
& m_{1}=0 \\
& m_{2}=c_{2}
\end{aligned}
$$

where $c_{1}$ and $c_{2}$ are integral constants. Finally, substituting (3.18) into (3.14), we get the result.

Corollary 3.3. Let $x: I \subset \mathbb{R} \rightarrow \mathbb{E}^{3}$ be a unit speed anti-Salkowski Tz-curve in $\mathbb{E}^{3}$ (with curvatures $\kappa_{1}>0$ and $\kappa_{2} \neq 0$ ) given with the parametrization (3.14). Then $x$ is congruent to $N$-constant curve of second kind.

Corollary 3.4. Let $x: I \subset \mathbb{R} \rightarrow \mathbb{E}^{3}$ be a unit speed Salkowski Tz-curve in $\mathbb{E}^{3}$ (with the curvatures $\kappa_{1}>0$ and $\kappa_{2} \neq 0$ ) given with the parametrization (3.14). Then we have

$$
m_{1}^{\prime \prime}+\left(\kappa_{1}^{2}+3 \kappa_{2}^{2}\right) m_{1}+\kappa_{1}=0
$$

where the curvature $\kappa_{1}$ of $x$ is a real constant.

Proof. Let $x$ be a unit speed Salkowski Tz-curve in $\mathbb{E}^{3}$. Hence, the curvature $\kappa_{1}$ of $x$ is constant, from the equation (3.15), we get the result.

Corollary 3.5. There is no Tz-curve with a constant curvature and a constant torsion. (i.e. Tz-W-curve)

Proof. Let $x$ be a unit speed Tz-curve in $\mathbb{E}^{3}$ with a constant curvature and a constant torsion. (i. e. Tz-W-curve). Then, using (3.15), we obtain

$$
\frac{\kappa_{1}(s)}{\kappa_{2}(s)}=\frac{c_{2}}{s+c_{1}}
$$

which is a contradiction. 


\section{R E F E R E N C E S}

1. M.E. Aydin, M. Ergüt: Non-null curves of Tzitzeica type in Minkowski 3-space. Romanian J. of Math. and Comp. Science 4(1) (2014), 81-90.

2. N. BiLA: Symmetry raductions for the Tzitzeica curve equation. Math. and Comp. Sci. Workin Papers 16 (2012).

3. A. Bobe, W. G. Boskoff And M. G. Ciuca: Tzitzeica type centro-affine invariants in Minkowski space. An. St. Univ. Ovidius Constanta 20(2) (2012), 27-34.

4. B. Y. Chen: Geometry of warped products as Riemannian submanifolds and related problems. Soochow J. Math. 28 (2002), 125-156.

5. B. Y. Chen: Convolution of Riemannian manifolds and its applications. Bull. Aust. Math. Soc. 66 (2002), 177-191.

6. B.Y. CHEN: When does the position vector of a space curve always lies in its rectifying plane?. Amer. Math. Monthly 110 (2003), 147-152.

7. M. CRAŞMAREAnN: Cylindrical Tzitzeica curves implies forced harmonic oscillators. Balkan J. of Geom. and Its App. 7(1) (2002), 37-42.

8. O. Constantinescu, M. Craşmareann.: A new Tzitzeica hypersurface and cubic Finslerian metrics of Berwall type Balkan J. of Geom. and Its App. 16(2) (2011), 27-34.

9. A. GRAY: Modern differential geometry of curves and surface, CRS Press, Inc. 1993.

10. H. GLuCK: Higher curvatures of curves in Euclidean space Amer. Math. Monthly 73 (1966), 699-704.

11. S. Gürpinar, K. Arslan, G. Öztürk: A Characterization of Constant-ratio Curves in Euclidean 3-space $E^{3}$. Acta Universitatis Apulensis 44 (2015), 39-51.

12. M. K. KARACAN, B. BüKÇÜ: On the elliptic cylindrical Tzitzeica curves in Minkowski 3-space. Sci. Manga 5 (2009), 44-48.

13. B. Kiliç, K. Arslan and G. Öztürk: Tangentially cubic curves in Euclidean spaces. Differential Geometry-Dynamical Systems 10 (2008), 186-196.

14. F. KLEIN, S. LIE: Uber diejenigen ebenenen kurven welche durch ein geschlossenes system von einfach unendlich vielen vartauschbaren linearen Transformationen in sich übergehen Math. Ann. 4 (1871), 50-84.

15. J. Monterde: Salkowski curves revisited: A family of curves with constant curvature and non-constant torsion. Computer Aided Geometric Design. 26 (2009) 271-278.

16. G. Öztürk, K. Arslan and H. HacisalihoĞLu: A characterization of ccrcurves in $\mathbb{R}^{n}$. Proc. Estonian Acad. Sciences 57 (2008), 217-224.

17. E. SALKOWSKI: Zur transformation von raumkurven. Mathematische Annalen. 66(4) (1909) 517-557.

18. G. E. VILCU: A geometric perspective on the generalized Cobb-Douglas production function. Appl. Math. Lett. 24 (2011), 777-783. 
Bengü Bayram, Emrah Tunç

Department of Mathematics

Balıkesir University

Balıkesir, TURKEY

benguk@balikesir.edu.tr, emrahtunc172@gmail.com

Kadri Arslan

Uludă̆ University

Department of Mathematics

Bursa, TURKEY

arslan@uludag.edu.tr

Günay Öztürk

Izmir Democracy University

Department of Mathematics

Izmir, TURKEY

gunay.ozturk@idu.edu.tr 